Telehealth Transition in a Comprehensive Care Unit for Eating Disorders: Challenges and Long-Term Benefits

Nandini Datta, PhD 1 *, Jennifer Derenne, MD1, Mary Sanders, PhD1, \& James D. Lock, MD, $\mathrm{PhD}_{1}$

1Department of Psychiatry and Behavioral Sciences

Stanford University School of Medicine

401 Quarry Road, Stanford CA, 94305

*Correspondence: Nandini Datta, Ph.D., Stanford University School of Medicine 401 Quarry Road, Stanford CA, 94305 nandinid@stanford.edu Abstract Word Count: 150

Manuscript Word Count: 3311 


\section{Acknowledgements and Conflicts of Interest}

Funding: The authors have no funding to disclose.

Conflict of Interest: The authors have no conflict to declare. 
Abstract

The COVID-19 pandemic has forced many eating disorder medical stabilization units

3 globally to consider creative adjustments that uphold both the quality of care delivered to

4 patients while also observing social distancing public health directives for patients and staff

5 alike. To date, inpatient facilities for eating disorders (both medical stabilization units and higher

6 level of care facilities) have not needed to consider how to translate services to electronic

7 platforms, given that most of these programs have in-person staff. We outline our transition to

8 telehealth broadly, emphasizing some unexpected benefits of using telehealth services that we

9 plan on integrating into our work-flow post COVID-19. These may be useful for other higher

10 level of care eating disorder programs, including medical stabilization units, residential, partial

11 hospitalization (PHP), and intensive outpatient programs (IOP). We also highlight aspects of

12 transition that have been more challenging for this particular patient population, warranting the

13 need for in-person services.

14 Keywords: Telehealth, Telesupervision, Eating Disorders, Inpatients, Adolescent, Psychiatry,

15 COVID-19, Anorexia Nervosa

16 
Telehealth Transition in a Comprehensive Care Unit for Eating Disorders: Challenges and Long-Term Benefits

Eating disorders (ED) are debilitating illnesses, characterized by a range of dysfunctional eating behaviors. These behaviors can result in severe medical complications requiring hospitalization. Eating disorders have the highest mortality rate of any mental health illness (Morris \& Twaddle, 2007), and when indicated by medical instability in the context of eating disorder behaviors, require comprehensive medical care and psychiatric support during hospitalization. Ideally, this is done by a multidisciplinary team of experts in eating disorders and ancillary staff. The utility of telehealth for EDs has previously been less explored than in-person evidence-based treatments, and has been identified as requiring further investigation to determine efficacy (Anderson, Byrne, Crosby, \& Le Grange, 2017; Kazdin, Fitzsimmons-Craft, \& Wilfley, 2017).

The coronavirus disease pandemic has resulted in a sudden transition to remote services, forcing clinicians to consider how to flexibly transfer outpatient treatment modalities to delivery via videoconferencing platforms. Remote delivery of Cognitive Behavioral Therapy for eating disorders (CBT-ED) and Family Based Treatment (FBT) have been recently discussed (Matheson, Bohon, \& Lock, 2020; Waller et al., 2020) to guide clinicians in this quick transition to remote services. Cooper et al. (2020) review literature pertinent to remote outpatient services for patients with eating disorders, highlighting unique challenges this population may be experiencing in light of the pandemic. What remains to be explored is the transition of inpatient services to telehealth for more acute patients with eating disorders.

Inpatient services transitioning to telehealth are understandably understudied, given that most hospital units have access to in-person clinicians. The COVID-19 public health crisis has 
1 forced ED care units globally to consider how to best support staff and patients, while observing

2 social distancing and minimizing in-person contact to mitigate the spread of the virus. Touyz,

3 Lacey, and Hay (2020) highlight the need for alternate methods of delivering care for

4 comprehensive care units in light of the COVID-19 pandemic; a recent article by Davis et al.,

5 (2020) outlines how a team in Singapore shifted to telehealth services for both an ED pediatric

6 inpatient unit and ED outpatient services in response to COVID-19. Our transition to inpatient

7 telehealth for psychiatry services has required similar consideration of factors outlined in this

8 paper in addition to those outlined in Weissman, Bauer, and Thomas (2020), ultimately

9 promoting maintenance of the same quality of care to patients while facilitating strong

10 communication and support amongst team members.

11 The Lucile Packard Children's Hospital Stanford's comprehensive care unit for eating

12 disorders psychiatry/psychology service transitioned to telehealth using guiding principles

13 outlined by Myers et al. (2017), including adherence to best practices, consideration of legal and

14 regulatory issues, upholding Health Insurance Portability and Accountability Act (HIPAA)

15 compliance, and consideration of available technology options. We outline the evolution of our

16 transition and highlight useful discoveries that we plan to incorporate post-COVID-19.

\section{Clinical Activities Impacted by COVID-19 Transition to Telehealth}

18 Our multidisciplinary eating disorder unit provides inpatient medical stabilization for

19 children and adolescents up to age 25 , admitting patients with medical consequences of

20 malnutrition, including bradycardia, orthostasis, hypotension, and severely low weight $(<75 \%$

21 median Body Mass Index). In transitioning to telehealth, all patient face-to-face interactions and

22 professional interactions with team members involve adherence to the following guidelines that 
1 have been discussed in a recent article regarding remote outpatient eating disorder treatment

2 (Waller et al., 2020) in addition to guidelines specific to inpatient work.

3 First, the space used for therapeutic sessions/intakes/groups/supervision (in the home)

4 must be private and professional in nature (from both the patient and therapist's perspective),

5 with therapist's credentialed badges visible. Second, measures should be taken prior to starting

6 sessions to ensure outside noise (pets, children, hospital staff, parents, etc.) and other distractions

7 are not interfering. Connectivity, hardware and software issues should be discussed beforehand,

8 and efforts should be made to ensure the patient has a working device to connect to remote

9 sessions. Hospital provided iPads are distributed to patients who do not have their own devices.

10 Lastly, all notes should include a phrase referencing patient consent obtained to proceed with

11 their visit over telehealth.

12 New Admission Evaluations

13 The psychiatric portion of intakes involves speaking with both the patient and parents.

14 Clinicians gather the history of ED behaviors to formulate a diagnosis and case

15 conceptualization. This includes decisions to implement 1:1 monitoring by nursing staff for

16 safety concerns or observation for two hours after meals to prevent engagement in compensatory

17 behaviors.

In the context of COVID-19, clinicians have utilized "Zoom," a video conferencing

19 platform, to complete intakes (Table 1). To do this, a Zoom link is created by the clinician and

20 distributed electronically to patients/parents via Epic MyChart messaging (a secure healthcare

21 messaging portal). When ancillary support is needed for coordination, this link is sent to nursing

22 staff via Voalte, a secure messaging portal for hospital employees (see Interdisciplinary

23 Communication). In the event a translator is required, clinicians either use an over-the-phone 
1 interpretative service available for a variety of languages or share the Zoom link with an

2 interpreter directly. Using interpreters and video conferencing has not appeared to impede

3 rapport building, obtaining history, and the overall efficacy of the intake session. Challenges

4 include a significant increase in time to schedule when these intakes will occur. Lastly, upon

5 admission parents receive a copy of "Help Your Teenager Beat an Eating Disorder" (J. Lock, Le

6 Grange, \& Tocci, 2018) to help with psychoeducation around eating disorder symptoms. This

7 book is currently delivered by nursing staff or delivered as an eBook.

Post-COVID Take Away. The value and ease of coordinating intake evaluations in

9 person outweigh benefits derived from the transition to telehealth. Still, the use of online

10 platforms has been useful in exploring alternative means of troubleshooting intakes (for example,

11 providing a way to bring in family members remotely) that will be utilized post-COVID.

Psychotherapy and Medication Management Sessions

Patients are seen daily, allowing clinicians to lay the groundwork for treatment and

14 support of continued renourishment efforts after discharge. For many families, their child's

15 hospitalization is their first experience working with a therapist with eating disorder expertise.

16 An unexpected benefit of using video conferencing is that it can allow for clinical interactions to

17 uncover challenges that may not otherwise have come up. An example of this is captured with

18 "Sully:"

19 Sully is a 16 year old gender non-binary patient with restrictive anorexia nervosa (AN-R),

20 admitted for significant weight loss in an attempt to suppress menstruation and feminine

21 secondary sex characteristics. Sully was resistant to remote sessions with the psychiatry

22 team, insisting their laptop and phone did not have working video. However, they also

23 refused using hospital provided devices, emphasizing a preference for phone calls or 
texting. Upon further discussion, Sully revealed that they struggled with social anxiety and were too self-conscious to look at themselves on the screen. Sully was agreeable to turn on the video briefly each session and made a goal to work towards leaving the video on for incrementally longer periods of time. They also agreed to work on attending group sessions using exposure and response prevention techniques. Here, remote sessions helped provide an opportunity for gradually expanding Sully's comfort level and promoting exposures that were tolerable.

In addition to the importance of individual sessions on our unit, the mobilization of families and parental empowerment is essential to the recovery process (Dimitropoulos, Freeman, Lock, \& Le Grange, 2017). Working with staff on the unit help families start to learn effective strategies for nutrition rehabilitation. At times, due to work restraints, caring for siblings, and geographical challenges, family members are not all able to physically attend family sessions or meals on the unit. The use of Zoom has facilitated the involvement of all essential family members integral in renourishment efforts to begin the learning process while their child is still hospitalized.

There have also been significant challenges over the course of using remote sessions, highlighting how avoidance may play a role in evading sessions or interactions with clinicians.

Patients have not connected to sessions despite telling nursing staff that they have, or have turned off their video when reluctant to engage. Additionally, if sessions broach sensitive issues with parents or patients, it appears to be more difficult to schedule sessions and find common times to meet. Jointly, these challenges have highlighted the necessity for staff to return to in-person work with this particular patient population when safe to do so. 
Post-COVID Take Away. Despite obstacles that have occurred during telehealth

2 sessions, remote sessions have provided some benefits that will be useful post-COVID. These

3 include unique opportunities to provide desensitization to social anxiety triggers and similar

4 clinical problems, and to increase opportunities for families to learn how to support their child

5 eating upon discharge. Zoom will continue to be used for these purposes after the COVID

6 pandemic.

\section{$7 \quad$ Therapeutic Groups}

8 Therapeutic groups are integral for teaching concrete skills and giving patients

9 opportunities to learn from and support one another. Groups can be helpful platforms to obtain

10 peer support and minimize isolation but may also be challenging due to increased opportunities

11 for social comparisons. Since 'Tele-groups', or groups via video conferencing, allow only the

12 patient's face to be visible, the intensity of comparisons to others body shape and size is reduced.

13 In addition, clinicians have observed increased participation from group members since shifting

14 to Tele-groups that may be due to decreased social anxiety and threat since participants are not in

15 the same room. While ultimately it will be important for these anxieties to be addressed, in the

16 context of a short-term inpatient hospitalization, it may be reasonable to defer them and instead

17 focus on increasing verbal participation. A recent study on the impact of COVID-19 on eating

18 disorders found patients with $\mathrm{AN}$ noted a negative correlation between isolation and their mental

19 health; conversely, helping vulnerable others provided a sense of purpose for patients and their

20 caregivers (Fernandez-Aranda et al., 2020). This could help explain the increased participation

21 noticed in groups where patients and/or parents support one another. An added general benefit

22 for Tele-groups is the inclusion of patients who would otherwise be unable to join. 
"Ground rules" are reviewed at the onset of groups to ensure close adherence to

2 protocols: restriction of bathroom use during group time to prohibit purging or exercise, review

3 of confidentiality (checking that patients are alone in their rooms or using headphones when not,

4 placing "in session" signs on patient doors to limit interruptions), and requesting no other

5 activities occur concurrently. The clinician enables the waiting room function and disables the

6 record feature to further protect privacy and confidentiality. Specific examples of how group

7 work can be conducted remotely are with our weekly Cognitive Behavioral (CBT), Cognitive

8 Remediation (CRT), Process (PG) and Health Education (HE) group therapies.

9 CBT is a patient-only group covering psychoeducation and practice of skills to

10 externalize ED thoughts and cope with difficult aspects of hospitalization. CBT typically uses

11 paper-pen materials; upon shifting to telehealth, this group has utilized the "share screen"

12 function for disseminating materials and the "whiteboard" function to allow group members to

13 participate actively in group activities and see what others are writing (Figure 1, 2). CRT is a

14 patient-only group (Lock et al., 2013; Tchanturia \& Lock, 2011) that aims to help patients

15 practice cognitive flexibility through interactive games and discussion of real-life applicability.

16 In transitioning to telehealth, group materials and activities are delivered for collaborative

17 participation via "screen share." PG is a patient-only group that provides a safe space for patients

18 to discuss their hospital experience, ask questions, and provide peer support (Yalom, 1988).

19 Therapeutic concepts of instilling of hope, universality, imparting information, altruism, and

20 socialization are emphasized (Yalom, 1995). While this group can often be met with reluctance

21 to participate, since transitioning to remote delivery, clinicians have observed increased

22 reciprocal conversation amongst patients. Since patients have been isolated in their rooms during

23 the COVID pandemic, PG provides a rare opportunity to share struggles and connect, which may 
1 contribute to increased willingness to participate. Lastly, HE groups are offered for patients and

2 parents to provide education about EDs and different treatment approaches, with a particular

3 focus on FBT, stages of change, relapse prevention, and discharge planning. HE Tele-groups

4 have used PowerPoint slides to relay information and facilitate discussion. Slides can be sent

5 directly to the family for ongoing reference. An unanticipated benefit is that parents who are at

6 home during the group time may also participate using the Zoom link, which hasn't been

7 possible during in-person sessions.

8 One of the downsides of the telehealth groups is that patients may have technical

9 problems logging into sessions or may feign these issues if they are hesitant or do not wish to

10 attend the group. If a patient is particularly resistant to attending treatment session, this may be

11 better handled in person rather than remotely.

12 Post-COVID Take Away. The clinical benefits of having in-person groups outweigh the

13 convenience of telehealth groups; however, there are a few aspects of remote groups we plan on

14 continuing to use. Zoom will be used to include patients who are on strict bed rest or isolation

15 and to involve family members who are not at the unit in person. Additionally, the delivery of

16 Tele-group materials to promote collaboration has been helpful in increasing participation,

17 similar strategies will be incorporated post-COVID.

18 Impact of Telehealth on Interdisciplinary Team Work

19 Interdisciplinary Communication

20 In order to communicate important information learned about patients more urgently, our

21 team has utilized Voalte for texting or calling staff. Voalte is a HIPAA compliant healthcare text

22 messaging platform used to streamline communication between members of our team. One 
1 example of how Voalte has been integral in facilitating what may otherwise have been a rocky

2 intake is captured in "Ali's" admission:

Ali is a 20 year old female with 6-year history of AN-R, readmitted to our unit for nutritional rehabilitation in the context of restrictive eating and excessive exercise. Ali vocalized shame and guilt around repeated admissions. She also expressed anger about being asked similar questions by multiple providers during the intake process. In order to help Ali while still obtaining the necessary information needed to form a diagnoses, the clinician connected with the providers who had already seen Ali (via Voalte) to obtain the requisite information they had gathered. With this data in hand, the psychologist met with Ali, confirmed the information obtained, asked if there was any other information Ali felt might be important to share, and dedicated remaining time to introducing coping skills. Ali expressed appreciation for this streamlined approach.

Using Voalte has facilitated communication among team-members; during the work-day, it is often difficult to catch providers in person. Downsides include the fact that team members sometimes feel hesitant to reach out via technology for fear that they were disturbing the clinician or because they have not been used to doing so.

Another specific example of remote interdisciplinary communication is during patient team rounding. Rounds are held every morning with all relevant members of our rich multidisciplinary team. Each patient is discussed to provide comprehensive updates on medical, nutritional, and behavioral status. Since transitioning to telehealth, members of the team working remotely connect to "Tele-rounds" with Zoom, and those physically present connect from their individual offices in order to observe appropriate social distancing practices. An addition to the daily schedule in light of the COVID-19 crisis has been an end of day clinical wrap up and 
1 "wellness rounds" which provides another opportunity for staff to gather for questions regarding

2 patient care and to help support one another.

Post-COVID Take Away. Care coordination exclusively over telehealth has had some

4 obstacles, for example, scheduling to meet with families becomes more arduous when not

5 physically present. Additionally, remote work requires the team to adapt to new routines which

6 can be challenging. Yet, having to rapidly shift to remote services has yielded some benefits that

7 we will continue to use post-COVID. These include the use of Voalte for quick interdisciplinary

8 communication between hospital staff and remote rounding to allow team members who need to

9 be physically away from the unit to participate in multidisciplinary team discussion.

\section{Training and Supervision}

11 One of the greatest benefits of using video conferencing platforms for supervision ("Tele-

12 supervision") is the ability to meet when the supervisor and trainee are not in the same location.

13 Additionally, the use of video-platforms with encrypted recording capabilities has increased the

14 ease with which supervisors can review recorded sessions with trainees or join sessions remotely.

15 Group supervision and staffing after rounds has utilized split screen features to involve all

16 trainees despite being in a variety of locations. Thus far, literature has found in-person and Tele-

17 supervision to be equivalent in establishing rapport and management of clinical issues (Jordan \&

18 Shearer, 2019). Broadly, ensuring proper orientation to and clear instruction of Tele-supervision

19 is important for success. Being siloed eliminates the ease of obtaining "supervision on the fly";

20 to remedy this, supervisors provide easy and quick access (secure text, Voalte messages,

21 encrypted email, calls) to trainees.

Post-COVID Take Away. Tele-supervision has been incredibly useful during this time

23 and will be used post-COVID as an equally effective and far more convenient alternative to 
1 supervision in person. Additionally, the use of Tele-supervision to allow remote connection to

2 sessions or encrypted recording will be used post-COVID for training purposes.

\section{Conclusions: Changes to Stay}

4 In sum, we have found the use of technology, flexibility among team-members, and

5 emphasis on self-care and communication to be integral in our efforts to provide high-level care

6 to our patients during the COVID-19 pandemic. While the majority of services provided are

7 conducted more easily when in-person, several of the COVID remote procedures had the

8 surprising benefit of utility post-COVID restrictions -- for team cohesion, patient care, and

9 training.

For team cohesion, the current COVID crisis has required creativity to facilitate

11 openness and communication amongst team members. Even in-person, this is often a challenge

12 with varying schedules and meetings. Ongoing use of Voalte will be useful to help facilitate

13 consultation on-the-fly. Additionally, remote access to meetings will extend benefits post-

14 COVID by allowing team members to contribute to patient care meetings, rounds, and care

15 conferences when they are at different locations. Remote conferencing-in specialists will allow

16 for medical consultation with experts who are not on site.

17 For patient care, we have learned that Tele-groups help include patients who otherwise

18 would not be able to join. Ongoing use of Zoom to include these patients will be useful post-

19 COVID to enhance patient care from the start of admission. Another benefit of remote sessions

20 includes allowing family members restricted by geographical reasons to participate in family

21 meals and sessions. Now, family members can more easily attend sessions, visit with their

22 children, and consult with the staff. Ongoing use of remote sessions to bring in all family

23 members will be utilized post-COVID. 
For training, Tele-supervision has been a viable alternative to in-person supervision:

2 reducing travel costs, increasing accessibility, and providing enhanced training opportunities.

3 Ongoing use of Tele-supervision will be effective in reducing travel burdens and improving

4 accessibility for trainees and supervisors. Similarly, our team has used video conferencing for

5 remote didactics with experts in ED outpatient treatments and attendance of Grand Rounds.

6 These will continue to provide utility for accessing material taught by experts in the field while

7 minimizing scheduling impacts.

8 These benefits may extend to other ED services both during navigation of transition to

9 telehealth and integration into the standard practice of care thereafter. PHP, IOP, and residential

10 programs may benefit from remote sessions to involve family members more easily, for

11 involvement in care and consultation with providers. Additionally, remote services may prove

12 useful in consultation with community providers during referral placement or discharge

13 planning. Remote services may help ED programs access resources and consultations from

14 specialists outside of their practice, contributing to the dissemination of treatment research across

15 levels of care. While the inevitable challenges faced during this rapid transition have highlighted

16 the importance of in-person work with ED patients, we hope incorporation of the aforementioned

17 benefits of remote work will continue to improve the quality of care moving forward. 


\section{References}

Anderson, K. E., Byrne, C. E., Crosby, R. D., \& Le Grange, D. (2017). Utilizing Telehealth to deliver family-based treatment for adolescent anorexia nervosa. International Journal of Eating Disorders, 50(10), 1235-1238. doi:10.1002/eat.22759

Cooper, M., Reilly, E. E., Siegel, J. A., Coniglio, K., Sadeh-Sharvit, S., Pisetsky, E., \& Anderson, L. (2020). Eating disorders during the COVID-19 pandemic: An overview of risks and recommendations for treatment and early intervention. https://doi.org/10.31234/osf.io/x7hea. doi: https://doi.org/10.31234/osf.io/x7hea

Dimitropoulos, G., Freeman, V. E., Lock, J., \& Le Grange, D. (2017). Clinician perspective on parental empowerment in family-based treatment for adolescent anorexia nervosa. Journal of Family Therapy, 39(4), 537-562. doi:10.1111/1467-6427.12086

Fernandez-Aranda, F., Casas, M., Claes, L., Bryan, D. C., Favaro, A., Granero, R., .. . Treasure, J. (2020). COVID-19 and implications for eating disorders. European Eating Disorders Review, 28(3), 239-245. doi:10.1002/erv.2738

Jordan, S. E., \& Shearer, E. M. (2019). An exploration of supervision delivered via clinical video telehealth (CVT). Training and Education in Professional Psychology Training and Education in Professional Psychology, 13(4), 323-330.

Kazdin, A. E., Fitzsimmons-Craft, E. E., \& Wilfley, D. E. (2017). Addressing critical gaps in the treatment of eating disorders. International Journal of Eating Disorders, 50(3), 170-189. doi:10.1002/eat.22670

Lock, Agras, W. S., Fitzpatrick, K. K., Bryson, S. W., Jo, B., \& Tchanturia, K. (2013). Is outpatient cognitive remediation therapy feasible to use in randomized clinical trials for 
anorexia nervosa? International Journal of Eating Disorders, 46(6), 567-575. doi:10.1002/eat.22134

Lock, J., Le Grange, D., \& Tocci, N. (2018). Help Your Teenager Beat an Eating Disorder. Retrieved from https://www.overdrive.com/search?q=5EC6F184-8BF1-4DC3-9D4FAB9951D30B2D

Matheson, B., Bohon, C., \& Lock, J. (2020). Family-based Treatment via videoconferencing: Clinical recommendations for treatment providers during COVID-19 and beyond. 10.31234/osf.io/b3hcs. doi:10.31234/osf.io/b3hcs

Morris, J., \& Twaddle, S. (2007). Anorexia nervosa. Bmj-British Medical Journal, 334(7599), 894-898. doi:10.1136/bmj.39171.616840.BE

Myers, K., Nelson, E.-L., Rabinowitz, T., Hilty, D., Baker, D., Barnwell, S. S., . . Bernard, J. (2017). American Telemedicine Association Practice Guidelines for Telemental Health with Children and Adolescents. Telemedicine and e-Health Telemedicine and e-Health, 23(10), 779-804.

Tchanturia, K., \& Lock, J. (2011). Cognitive Remediation Therapy for Eating Disorders: Development, Refinement and Future Directions. In R. A. H. Adan \& W. H. Kaye (Eds.), Behavioral Neurobiology of Eating Disorders (Vol. 6, pp. 269-287).

Touyz, S., Lacey, H., \& Hay, P. (2020). Eating disorders in the time of COVID-19. Journal of Eating Disorders, 8(1). doi:10.1186/s40337-020-00295-3

Waller, G., Pugh, M., Mulkens, S., Moore, E., Mountford, V. A., Carter, J., . . Smit, V. (2020). Cognitive-behavioral therapy in the time of coronavirus: Clinician tips for working with eating disorders via telehealth when face-to-face meetings are not possible. International Journal of Eating Disorders. doi:10.1002/eat.23289 
Weissman, R. S., Bauer, S., \& Thomas, J. J. (2020). Access to evidence-based care for eating disorders during the COVID-19 crisis. International Journal of Eating Disorders. doi:10.1002/eat.23279

Yalom, I. D. (1988). Inpatient group psychotherapy. New York, N.Y.: Basic Books.

Yalom, I. D. (1995). Theory and practice of group psychotherapy. BasicBooks: N. Y. 


\section{Figure Legend}

Figure 1. During groups, this is the clinicians screen. "Participant 2" is in the virtual waiting room, not yet admitted. Participants 1,3 and 4 have been admitted. The "clinician" is using group view to see all members simultaneously.

Figure 2. During screen share from the clinician, this is the participant's view of the screen and options to participate collaboratively on the bottom (see: annotate). 